\title{
Variabilidade da frequência cardíaca na recuperação pós-exercício máximo em jovens sobrepesos
}

http://dx.doi.org/10.11606/1807-5509201800030329

\author{
Gabriel Kolesny TRICOT* \\ Jaqueline Alves de ARAÚJO* \\ Gisela ARSA* \\ Lucieli Teresa CAMBRI* \\ *Departamento de \\ Educação Física, \\ Universidade Federal \\ de Mato Grosso, \\ Cuiabá, MT, Brasil
}

\section{Resumo}

0 objetivo deste estudo foi analisar a variabilidade da frequência cardíaca (VFC) na recuperação ativa em jovens com diferentes índices de massa corporal (IMC) e associar as variáveis em repouso e recuperação. Dezoito jovens, eutróficos - E $\left(22,36 \pm 1,92 \mathrm{~kg} \cdot \mathrm{m}^{-2}\right)$ e sobrepesos $-\mathrm{S}\left(29,71 \pm 2,97 \mathrm{~kg} \cdot \mathrm{m}^{-2}\right)$, foram avaliados. A frequência cardiaca (FC) e os índices de VFC (RMSSD e SDNN) em repouso, exercício e na recuperação após exercício máximo durante 5 minutos, assim como, as áreas sob a curva da $\mathrm{VFC}$, foram analisadas. 0 consumo máximo de oxigênio - $\mathrm{VO}_{2 \text { máx }}$ foi menor $(\mathrm{p}<0,05)$ nos jovens sobrepesos. Os índices $\mathrm{LnRMSSD}_{30 \mathrm{~s}} \mathrm{e}$ $\operatorname{LnSDNN}_{30 \mathrm{~s}}$ ao final do exercício ( $\mathrm{E}: 0,46 \pm 0,11$ e 0,75 $\pm 0,12 ; \mathrm{S}: 0,58 \pm 1,15$ e 0,73 \pm 0,12 ms) estão reduzidos em relação ao repouso ( $\mathrm{E}: 1,56 \pm 0,11$ e 1,72 $\pm 0,13$; $\mathrm{S}: 1,42 \pm 0,11$ e 1,65 $\pm 0,23 \mathrm{~ms})$, e permaneceram inferiores $(p<0,05)$ ao final da recuperação (E: 0,54 $\pm 0,23$ e 0,67 $\pm 0,24 ; S: 0,47 \pm 0,23$ e 0,67 $\pm 0,23$ ms) sem diferença entre os grupos. Além disso, as variáveis morfológicas associam-se $(p<0,05)$ à aptidão aeróbia ( $r=-0,71$ a -0,76), e a FC aos índices da VFC nos diferentes momentos fisiológicos. Em conclusão, não há reativação vagal após 5 minutos de recuperação ativa de um exercício máximo em jovens, com comportamento similar entre eutróficos e sobrepesos. Igualmente, as variáveis morfológicas associam-se inversamente à aptidão aeróbia, e a FC aos índices de VFC nos diferentes momentos fisiológicos.

Palavras-Chave: Obesidade; Modulação autonômica cardiaca; Aptidão aeróbia.

\section{Introdução}

O monitoramento da frequência cardíaca (FC) e de sua variabilidade (VFC) são parâmetros importantes na avaliação do sistema nervoso autonômico (SNA) cardíaco em diferentes condiçóes de saúde ${ }^{1,2}$, sobretudo, por ser uma medida simples, não invasiva, de baixo custo e de alta praticidade clínica, que pode ser obtida com o uso de cardiofrequencímetros ${ }^{3,4}$. A partir de métodos lineares analisados no domínio do tempo, são obtidos os índices da VFC, que refletem atuação do ramo parassimpático e interação dos ramos simpático e parassimpático da modulação autonômica cardíaca, sendo estes, respectivamente, a raiz quadrada da média das diferenças sucessivas ao quadrado, entre intervalos R-R adjacentes (RMSSD) e o desvio padrão de todos os intervalos R-R normais gravados em um intervalo de tempo (SDNN) $)^{4}$.

Os mecanismos envolvidos no comportamento da FC e da VFC durante o repouso estáo bem estabelecidos, sendo que doenças como a obesidade $^{1,5}$, hipertensão ${ }^{1}$, e diabetes melitus ${ }^{2}$, acarretam prejuízos na modulação autonômica cardíaca, com maior atuação do ramo simpático e redução dos índices da VFC associados ao sistema nervoso parassimpático ${ }^{4}$. Adicionalmente, outros fatores como idade ${ }^{6,7}$, posiçáo corporal ${ }^{8} \mathrm{e}$ aptidão aeróbia ${ }^{3}$ podem influenciar na modulaçáo autonômica cardíaca.

Durante o exercício físico, ocorrem adaptaçóes no SNA devido ao estresse fisiológico, assim, a FC aumenta no início do exercício, devido à diminuição da ati vidade parassimpática. Por sua vez, o ramo simpático é estimulado, provocando o aumento gradativo da FC e redução da VFC, até o momento em que se torna predominante, com a FC aumentando continuamente e a VFC tendendo a zero ${ }^{3}$. 
$\mathrm{Na}$ recuperação pós-exercício, a FC diminui rapidamente nos instantes iniciais, com boa reprodutibilidade em exercício realizado no cicloergômetro $^{9}$ e esteira rolante ${ }^{10}$, tanto em jovens, quanto em idosos ${ }^{11}$. Os mecanismos envolvidos e o comportamento da VFC neste momento fisiológico ainda são controversos. Nesse sentido, muitos estudos ${ }^{12-14}$ sugerem que a redução da FC nos primeiros minutos da recuperação é devido a reativação vagal e posteriormente a retirada simpática. Por outro lado, a retirada simpática, nos instantes iniciais da recuperação pós-exercício é igualmente apontada como ramo autonômico responsável pela redução da $\mathrm{FC}$, e posteriormente a reativação vaga ${ }^{15}$. Além disso, é bem estabelecido que, a FC de recuperação pós-exercício é forte preditora de mortalidade na população em geral ${ }^{12-14}$.

A FC e a VFC são utilizadas como indicadores de aptidão aeróbia, também associadas a condiçôes favoráveis e desfavoráveis à saúde. Além disso, podem ser utilizados tanto na prescrição de exercício físico, quanto no acompanhamento de adaptaçóes cardiovasculares em distintos momentos fisiológicos, como em repouso, exercício físico e recuperação pós-exercício ${ }^{1,3,12,15,16}$, nas mais variadas populaçōes ${ }^{1,2,4,5}$.

Contudo, as respostas do SNA durante a recuperação pós-exercício máximo, não estão completamente elucidadas, especialmente em jovens sobrepesos náo treinados. Uma vez que, é principalmente avaliada na estratificação dos riscos de morte e sobrevida após decorrências cardíacas, em indivíduos com diferentes doenças, assim como, em sujeitos fisicamente ativos ${ }^{15,16}$.

Neste sentido, a hipótese deste estudo é a de que não haja reativação vagal na fase inicial da recuperação após exercício máximo em jovens com baixa aptidão aeróbia, e que os jovens com excesso de massa corporal apresentam alteraçóes da modulação autonômica cardíaca.

Portanto, o presente estudo tem como objetivo analisar o comportamento da VFC na recuperação ativa em jovens com diferentes índices de massa corporal e verificar as associaçóes das variáveis referentes à modulação autonômica cardíaca em repouso e na recuperação.

\section{Métodos}

\section{Participantes}

Foram avaliados 18 universitários do sexo masculino, com idades entre 18 e 25 anos, aparentemente saudáveis, considerados não treinados pela ausência de práticas de exercício físico regular nos quatro meses que antecederam o início do estudo. a partir de amostragem não aleatória por conveniência. Destes, nove eutróficos (IMC $\leq 24.9 \mathrm{~kg} \cdot \mathrm{m}^{-2}$ ) e nove sobrepesos (IMC $\geq 25 \mathrm{~kg} \cdot \mathrm{m}^{-2}$ ). Os procedimentos do estudo foram aprovados pelo Comitê de Ética em Pesquisa sob o parecer de no 19109213.2.0000.5541 e todos os voluntários assinaram um Termo de Consentimento Livre Esclarecido. Os critérios de exclusão foram: fumar, uso de medicaçáo contínua, morte súbita de familiares de primeiro grau com menos de 40 anos, problemas articulares, obesidade mórbida e demais doenças.

\section{Procedimentos}

Os avaliados foram orientados a não praticar atividades vigorosas, e a não ingerir bebidas estimulantes nas 24 horas anteriores às avaliaçóes. Igualmente, foram aconselhados a realizar a última refeição habitual, duas horas antes do início das avaliaçóes.

Inicialmente, uma anamnese, composta por dados de identificação, relato de doenças e de histórico familiar foi aplicada. Caso o voluntário relatasse algum evento que pudesse oferecer risco à sua integridade durante as avaliaçóes, seria excluído do estudo, porém, isto não ocorreu com nenhum dos participantes.

O questionário internacional de atividade física (IPAQ), em sua versão curta, foi aplicado para classificar o nível de atividade física dos avaliados, em sedentário, irregularmente ativo, ativo e muito ativo. Os valores de equivalente metabólico (METs. minutos/semana) foram calculados pelo somatório para cada uma das dimensóes de atividade física avaliadas (caminhada, atividade moderada e vigorosa). Estas foram calculadas multiplicando-se a frequência (dias/semana), o tempo (minutos/dia) e a intensidade (METs) correspondente a cada uma das dimensões. Em seguida, foi calculado o volume de atividade semana por meio da soma do produto obtido em cada uma dessas multiplicaçóes. 
O índice de massa corporal (IMC) foi determinado pela razão massa corporal (balança CAMRY", $100 \mathrm{~g}$ ) e estatura elevada ao quadrado (estadiômetro SANNY, 0,1 cm).

A FC e VFC em repouso foram mensuradas utilizando um monitor de FC portátil (POLAR, RS800CX) com registros batimento a batimento, por meio dos intervalos R-R durante 10 minutos contínuos, com os indivíduos na posição sentada em cadeira estofada. Os cinco primeiros minutos foram descartados e a média da mensuraçáo dos cinco minutos finais de repouso foi considerada para análise.

Os intervalos R-R foram tratados no programa Kubios HRV, com os artefatos filtrados a nível moderado, e os índices de VFC utilizados foram os referentes ao domínio do tempo: raiz quadrada da média das diferenças sucessivas ao quadrado entre intervalos R-R adjacentes (RMSSD) e desvio padrão de todos os intervalos $\mathrm{R}-\mathrm{R}$ normais gravados em um intervalo de tempo (SDNN).

Anteriormente ao teste progressivo máximo, foram realizadas duas mensuraçóes da pressão arterial (MICROLIFE, BP3T0-A), com o indivíduo na posição sentada após 10 minutos de repouso e com dois minutos de intervalo entre cada mensuração. Quando observada diferença entre as mensurações foi realizada uma terceira e calculada a média entre as mesmas. Todos os jovens avaliados foram considerados normotensos $(<140 / 90 \mathrm{mmHg})$.

\section{Protocolo de exercício}

Foi realizado um teste progressivo máximo em cicloergômetro (INBRASPORT, CG-04), com carga inicial de 15 watts (w), acrescidos $15 \mathrm{w}$ a cada minuto, com manutenção de $60 \mathrm{rpm}$, até exaustão voluntária. Para considerar o teste como máximo, os avaliados deveriam atingir no mínimo $90 \%$ da FC máxima predita pela idade (220 - idade) e não serem capazes de manter $60 \mathrm{rpm}$.

\section{Resultados}

A TABELA 1 apresenta os valores de média e desvio padrão das variáveis morfofisiológicas no repouso.

Os jovens sobrepesos apresentaram valores superiores $(\mathrm{p}<0,05)$ de massa corporal e IMC. A FC
Quando o último estágio não foi completado, a carga máxima foi determinada pela equação: Carga $_{\text {máx }}=$ Carga no estágio completo $+[$ (tempo $(s)$ no último estágio/60) x 15]. Ao final do teste progressivo, a FC, VFC, e a relação carga máxima e massa corporal $\left(w . \mathrm{kg}^{-1}\right)$ foram determinadas. O período de recuperação foi realizado de forma ativa no cicloergômetro durante 5 minutos com $15 \mathrm{w}$, sendo identificados os valores de FC e dos índices RMSSD e SDNN da VFC a partir da média dos 5 minutos de recuperaçáo e a cada 30 segundos e posteriormente os dados da VFC foram transformados em logaritmos naturais (Ln). Adicionalmente, foram identificadas as áreas sob a curva da FC e dos índices da VFC, a partir dos valores de pico do exercício e recuperaçáo pós-exercício, obtidas pela equação: $\sum[($ Área $1+$ Área2+Área 3...) ], onde cada área: [(Base maior + Base menor) $\mathrm{x}$ altura] $/ 2$. Foi considerado 30 segundos para altura obtendo-se 10 áreas, posteriormente somadas e obtidas o valor da área total. Além disso, a aptidão aeróbia foi estimada pelo consumo máximo de oxigênio $\left(\mathrm{VO}_{2 \max }\right)$ pela equação: $\mathrm{VO}_{2 \text { máx }}\left(\mathrm{mL} \cdot \mathrm{kg} \cdot \mathrm{min}^{-1}\right)=(13 \mathrm{x}$ carga máxima em $\mathrm{w} /($ massa corporal em $\mathrm{kg}+3,5)$. Todas as avaliaçóes ocorreram no período vespertino entre 14 e 16 horas, com temperatura de $25,11 \pm 5,09^{\circ} \mathrm{C}$ e umidade relativa do ar de 43,28 $\pm 5,13 \%$.

\section{Análise estatística}

Foi utilizada estatística descritiva com determinação da média e desvio padrão. Para analisar a normalidade dos dados foi utilizado o teste Shapiro Wilk. Para comparaçáo dos grupos utilizou-se o teste T não pareado para dados paramétricos e o teste $\mathrm{U}$ de Mann Whitney para dados não paramétricos. Osíndices da VFC a cada $30 \mathrm{~s}$, durante a recuperação, foram comparados pela ANOVA One-way e post hoc de Tukey $(\mathrm{p}<0,05)$. Para determinar a relação entre as variáveis analisadas foram empregadas a correlação linear de Pearson e Spearman Rank's para dados paramétricos e não paramétricos, respectivamente. O nível de significância adotado foi de $5 \%(\mathrm{p}<0,05)$. e os índices RMSSD e SDNN em repouso não diferiram $(p>0,05)$ entre os grupos (TABELA 1$)$. O nível de atividade física em escores contínuos METS.minutos/semana, determinado pelo 
* $p<0,05$ entre os grupos pelo Teste- $T$ de Student's para dados paramétricos. METs foi a única variável analisada pelo Teste U de Mann Whitney para dados não paramétricos.
$\# p<0,05$ entre os grupos pelo Teste $U$ de Mann Whitney. para dados não paramétricos. As demais variáveis foram analisadas pelo teste T não pareado para dados paramétricos. RMSSD5rec: média do índice RMSSD nos 5 minutos de recuperação; $\mathrm{SDNN}_{5 \text { rec }}$ : média do índice SDNN nos 5 minutos de recuperação.
IPAQ não foi diferente entre os grupos ( $\mathrm{p}>0,05)$, e foram considerados $55,65 \%$ irregularmente ativos, $33,33 \%$ ativos e $11,11 \%$ muito ativos no grupo eutrófico, e 22,22\% irregularmente ativos e $77,78 \%$ ativo no grupo sobrepeso, conforme TABELA 2.

TABELA 1 - Média e desvio padrão das variáveis morfofisiológicas no repouso e nível de atividade física

\begin{tabular}{lcc}
\hline & Eutróficos $(\boldsymbol{n}=\mathbf{9})$ & Sobrepesos $(\boldsymbol{n}=\mathbf{9})$ \\
\hline Idade (anos) & $21,23 \pm 1,99$ & $21,66 \pm 1,91$ \\
Massa corporal (kg) & $67,24 \pm 7,29$ & $93,44 \pm 11,01^{*}$ \\
Estatura (m) & $1,73 \pm 0,08$ & $1,77 \pm 0,07$ \\
IMC (kg.m-2) & $22,36 \pm 1,92$ & $29,71 \pm 2,97^{*}$ \\
Frequência cardíaca (bpm) & $73,67 \pm 10,72$ & $77,50 \pm 13,65$ \\
RMSSDrepouso (ms) & $37,55 \pm 15,21$ & $32,81 \pm 24,60$ \\
SDNNrepouso (ms) & $55,37 \pm 17,99$ & $49,89 \pm 25,12$ \\
IPAQ Escore total (METs.min.sem-1) & $3545,00 \pm 5014,38$ & $1689,78 \pm 1143,79$ \\
\hline
\end{tabular}

TABELA 2 - Média e desvio padrão das variáveis de modulação autonômica cardíaca no teste progressivo máximo e na recuperação pós-exercício

\begin{tabular}{|c|c|c|}
\hline & Eutróficos $(n=9)$ & Sobrepesos $(n=9)$ \\
\hline Frequência cardíaca $_{\text {pico }}(\mathrm{bpm})$ & $195,78 \pm 11,34$ & $200,67 \pm 10,54$ \\
\hline Frequência cardíaca ${ }_{\text {máxima }}(\%)$ & $98,31 \pm 5,30$ & $100,94 \pm 4,99$ \\
\hline Cargamáxima (w) & $200,47 \pm 33,35$ & $213,19 \pm 19,09$ \\
\hline w.kg-1 ${ }^{1}$ & $2,98 \pm 4,58$ & $2,28 \pm 1,73 \#$ \\
\hline VO2máx (mL.kg.min-1) & $36,84 \pm 4,70$ & $28,76 \pm 2,91 \#$ \\
\hline RMSSD5rec (ms) & $3,17 \pm 1,00$ & $3,32 \pm 0,73$ \\
\hline SDNN5rec (ms) & $6,50 \pm 1,51$ & $5,28 \pm 1,68$ \\
\hline $\begin{array}{l}\text { Área sob a curva do LnRMSSD } \\
\left(\mathrm{ms} .300 \mathrm{~s}^{-1}\right)\end{array}$ & $127,76 \pm 38,16$ & $130,08 \pm 39,65$ \\
\hline Área sob a curva do LnSDNN (ms.300s- $\left.{ }^{1}\right)$ & $229,93 \pm 31,86$ & $201,35 \pm 39,22$ \\
\hline
\end{tabular}

O teste foi considerado máximo para todos os avaliados, uma vez que, os grupos eutrófico e sobrepeso atingiram $98,31 \pm 5,30$ e $100,94 \pm 4,99 \%$ da FC máxima predita para idade, respectivamente. A tabela 2 apresenta os valores de média e desvio padrão das variáveis de modulação autonômica cardíaca durante o teste progressivo máximo e na recuperação. Os jovens sobrepesos apresentaram a relação carga máxima/massa corporal $\left(\right.$ w. $\left.\mathrm{kg}^{-}{ }^{1}\right) \mathrm{e}$ $\mathrm{VO}_{2 \text { max }}$ inferiores $(\mathrm{p}<0,05)$ aos eutróficos. Quanto aos índices avaliados nos 5 minutos de recuperação, não foram observadas diferenças entre os grupos nas variáveis de RMSSD, SDNN e áreas sob a curva do LnRMSSD e LnSDNN.

A FC apresentou maiores valores nos sobrepesos, embora não estatisticamente significativos, comparados aos eutróficos, em todos os tempos da recuperação ativa pós-exercício (FIGURA 1).

As FIGURAS 2 e 3 apresentam a dinâmica dos índices LnRMSSD e LnSDNN no repouso, pico do exercício e o LnRMSSD e LnSDNN analisados a cada 30 segundos durante os cinco 
minutos após o exercício máximo. Em ambos os grupos, os índices LnRMSSD ${ }_{30 \mathrm{~s}}$ e $\mathrm{LnSDNN}_{30 \mathrm{~s}}$ refrentes ao pico do exercício reduziram $(\mathrm{p}<0,05)$ em relação ao repouso, e permaneceram reduzidos até final dos cinco minutos de recuperação ativa. Além disso, o índice $\mathrm{LnSDNN}_{30 \mathrm{~s}}$ nos 60 e 240 segundos da recuperação foi superior $(\mathrm{p}<0,05)$ no grupo eutrófico, comparado aos sobrepesos. A TABELA 3 apresenta as correlaçóes entre as variáveis referentes à modulação autonômica cardíaca no repouso e na recuperação.

A FC no repouso foi variável que mais se associou significativamente aos índices RMSSD $(\mathrm{r}=-0,57)$ e SDNN $(\mathrm{r}=-0,57)$ de repouso e com a área sob a curva do LnSDNN $(r=-0,57)$. Adicionalmente, observou-se associaçáo inversa $(\mathrm{p}<0,05)$ do $\mathrm{VO}_{2 \text { máx }}$ com a massa corporal $(\mathrm{r}=-0,71)$ e IMC $(\mathrm{r}=-0,72)$.

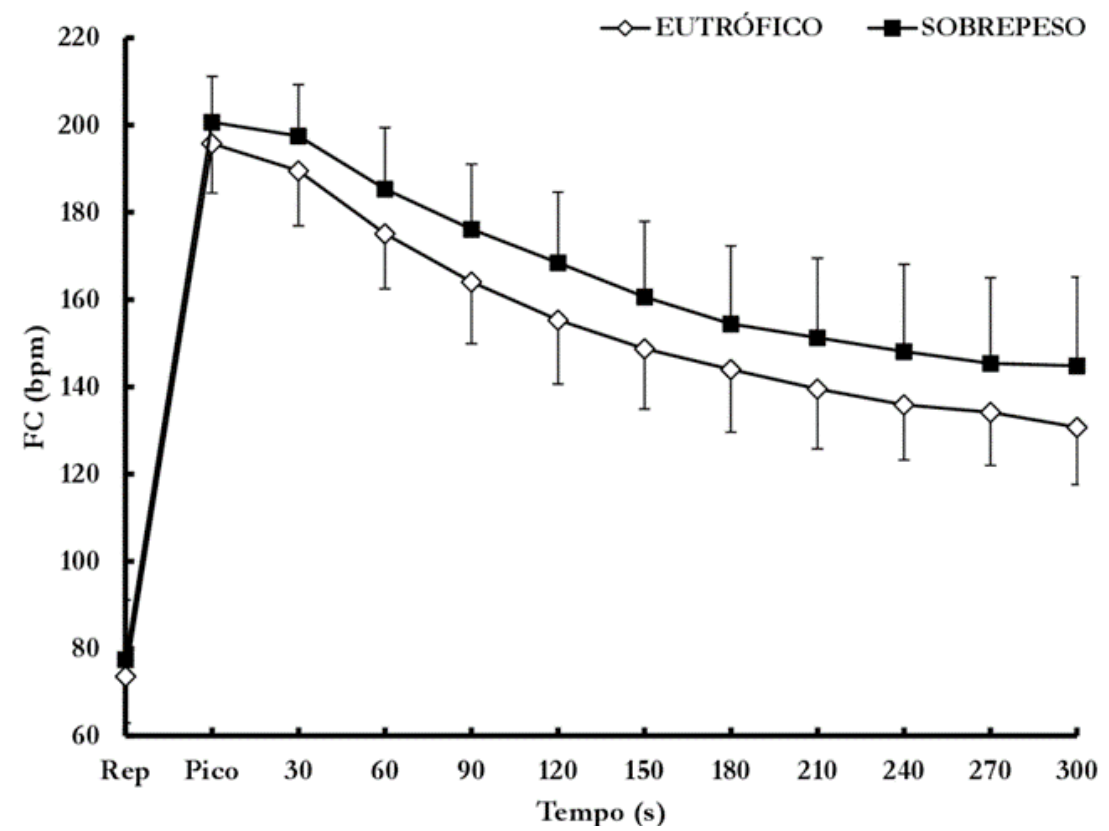

FIGURA 1 - Dinâmica da FC no repouso, pico do exercício e a cada 30 segundos após exercíco máximo

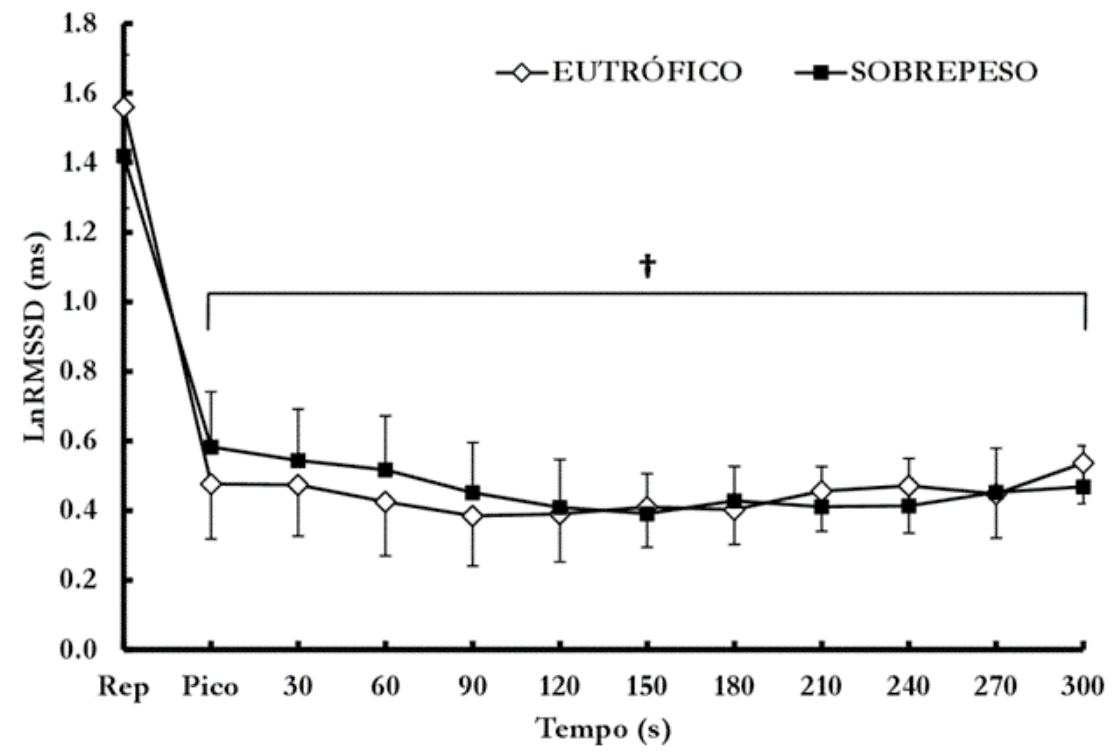

FIGURA 2 - Dinâmica do indice LnRMSSD no repouso, pico do exercício e LnRMSSD3os após exercíco máximo. ${ }^{\dagger} \mathrm{p}<0,05 \mathrm{em}$ relação ao repouso. 


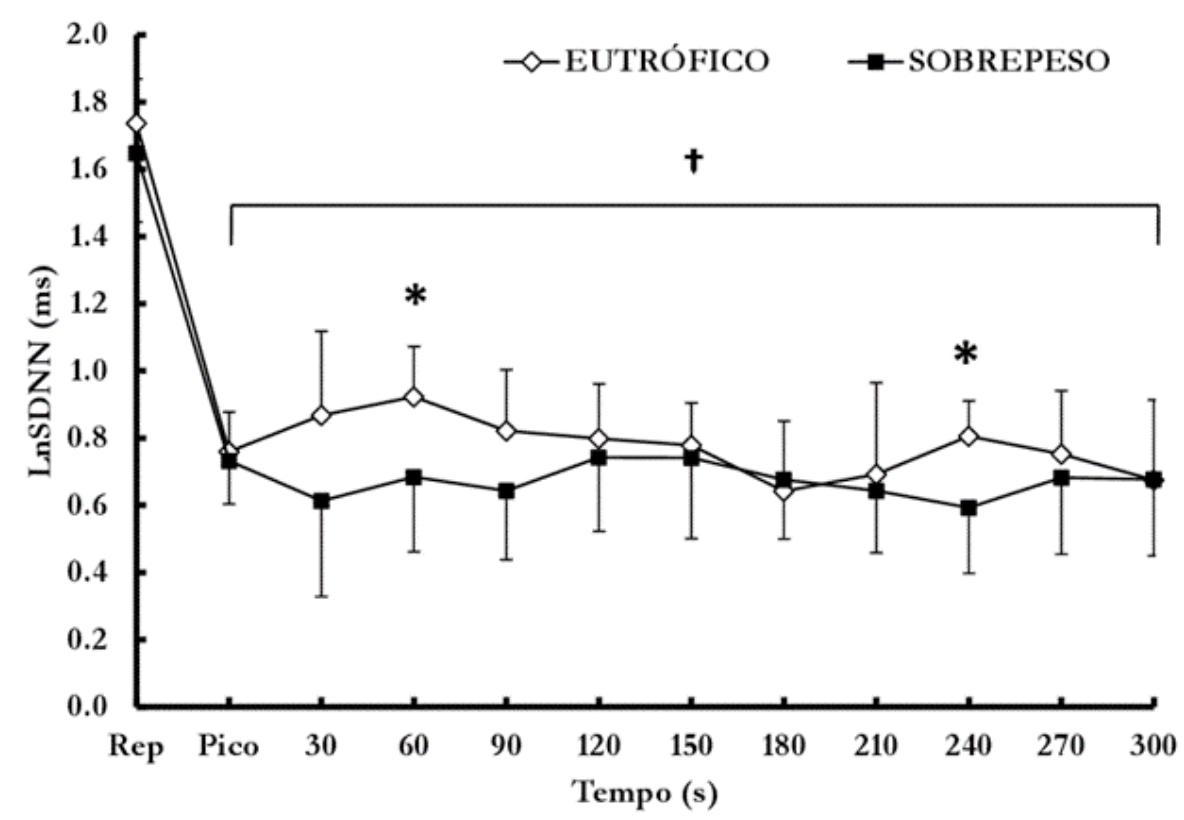

FIGURA 3 - Dinâmica do indice LnSDNN no repouso, pico do exercício e LnSDNN3os na recuperação após exercíco máximo. ${ }^{\dagger} \mathrm{p}<0,05$ em relação ao repouso. ${ }^{*} \mathrm{p}<0,05$ entre os grupos.

${ }^{*} p<0,05$ coeficiente de correlação linear de Pearson;

\# coeficiente de correlação linear de Spearman's

Rank. $\mathrm{FC}_{30 \mathrm{rec}}:$ média da frequência cardíaca nos 30 s de recuperação; $\mathrm{FC}_{\text {60rec }}$ média da frequência cardíaca nos 60 s de recuperação. $\mathrm{FC}_{30 \text { rec }}$ foi a única variável analisada por testes não paramétricos.

TABELA 3 - Correlação das variáveis referentes à modulação autonômica cardíaca

\begin{tabular}{lccc}
\hline $\mathbf{n}=\mathbf{1 8}$ & $\mathbf{F C}_{\text {repouso }}(\mathbf{b p m})$ & $\mathbf{F C}_{30 \mathrm{rec}}(\mathbf{b p m})$ & $\mathbf{F C}_{\text {60rec }}(\mathbf{b p m})$ \\
\hline $\operatorname{RMSSD}_{\text {repouso }}(\mathrm{ms})$ & $-0,57^{*}$ & $-0,29$ & $-0,29$ \\
$\operatorname{SDNN}_{\text {repouso }}(\mathrm{ms})$ & $-0,57^{*}$ & $-0,24$ & $-0,34$ \\
Área sob a curva do & $-0,46$ & $-0,56 \#$ & $-0,25$ \\
$\begin{array}{l}\left.\text { RMSSD (ms.300s- }{ }^{1}\right) \\
\text { Área sob a curva do }\end{array}$ & $-0,57^{*}$ & $-0,71 \#$ & $-0,70^{*}$ \\
SDNN (ms.300s- $\left.{ }^{-}\right)$ & & & \\
\hline
\end{tabular}

\section{Discussão}

Os principais resultados do presente estudo demonstram que os índices da VFC LnRMSSD ${ }_{30 s}$ e $\mathrm{LnSDNN}_{30 \mathrm{~s}}$ ao final de um exercício máximo estão reduzidos em relação ao repouso, e após 5 minutos de recuperação ativa continuam inferiores, com comportamento similar entre os grupos eutróficos e sobrepesos. Assim como, há associação entre a modulação autonômica cardíaca de repouso e a recuperação pós-exercício máximo.

A importância deste estudo se deve a avaliação de jovens sobrepesos, não treinados e saudáveis, uma vez que, a maioria dos estudos avaliam a modulação autonômica cardíaca em repouso e na recuperação em indivíduos de meia idade, com outras doenças já instaladas ${ }^{1,5}$ ou em indivíduos com desfechos cardiovasculares para avaliar risco de morte $^{11}$ e a chance de sobrevida pós eventos cardíacos ${ }^{12,13}$. Do mesmo modo, a modulação autonômica cardíaca na recuperação é avaliada, sobretudo, em jovens eutróficos não treinados e/ou fisicamente ativos ${ }^{15,16}$. O presente estudo é um dos primeiros a verificar a influência do excesso de massa corporal na reativação vagal pós-exercício máximo em jovens não treinados.

Alguns estudos relatam que a obesidade causa uma redução, tanto da atividade simpática, quanto da parassimpática em repouso ${ }^{5,17}$, com menores valores nos índices SD1, RMSSD, SDNN, pNN50 
e $\mathrm{HF}^{17,18}$, bem como, aumento dos valores do índice $\mathrm{LF}$ e da razão $\mathrm{LF} / \mathrm{HF}^{18} \mathrm{em}$ jovens obesos, quando comparados aos eutróficos ${ }^{17}$, o que náo foi observado no presente estudo.

Adicionalmente, neste estudo, o grupo sobrepeso apresentou valores inferiores de $\mathrm{VO}_{2 \text { máx }}$ quando comparado ao grupo eutrófico. Neste sentido, D’Agosto et al. ${ }^{19}$ demonstraram que indivíduos com maior aptidão aeróbia apresentam ajustes mais rápidos da VFC no início do exercício. Do mesmo modo, os níveis de aptidão aeróbia se associaram ao controle autonômico cardíaco na recuperação e maior reativação vagal pós-exercício em indivíduos entre 20 e $60 \operatorname{anos}^{7}$, sendo verificado risco de mortalidade cerca de sete vezes maior em indivíduos com baixa aptidão aeróbia e diminuída recuperação da FC, comparado à indivíduos com aptidão aeróbia e recuperação da $\mathrm{FC}$ elevados ${ }^{20}$.

Neste estudo, os avaliados, considerados não treinados, apresentaram nível de atividade física e carga máxima no teste progressivo semelhantes, independentemente do IMC. Sendo a diferença de $\mathrm{VO}_{2 \text { máx }}$ determinada pela diferença de massa corporal e confirmada pelas associaçóes inversas significantes do $\mathrm{VO}_{2 \text { máx }}$ com a massa corporal e com o IMC.

Ao final de cinco minutos de recuperaçáo ativa após exercíco máximo, os índices $\operatorname{LnRMSSD}_{30 \mathrm{~s}} \mathrm{e}$ $\mathrm{LnSDNN}_{30 \mathrm{~s}}$ mantiveram-se reduzidos em relação ao repouso e ao final do exercício, com comportamento similar entre os grupos, indicando a não reativação do sistema nervoso parassimpático. Corroborando o estudo de BARACK et al. ${ }^{8}$ com jovens sedentários, os índices RMSSD, SDNN e RRNN foram significativamente menores na recuperação passiva pós-exercício submáximo $(80 \% \mathrm{FC})$ em comparação aos valores de repouso, independentemente da posição corporal. O mesmo foi observado no índice RMSSD após exercício máximo e recuperação passiva por 5 minutos em jovens com maior capacidade aeróbia $^{15}$, e em jovens saudáveis sedentários ou em treinados em exercício de força ${ }^{16}$.

Em contrapartida, observou-se reativação vagal devido à elevação significativa do índice $\mathrm{RMSSD}_{30}$ entre os 210 e 300 segundos da recuperação nos indivíduos treinados aerobiamente ${ }^{16}$. Assim como, em jovens com melhor capacidade aeróbia do que os do presente estudo, o índice SDNN aumentou a partir de 90 segundos da recuperação, indicando que o mecanismo de controle responsável pela redução da FC na recuperação, nos instantes iniciais, estaria associado à retirada inicial da atuação do ramo simpático ${ }^{15}$. Estes fatos sugerem que a velocidade da reativação vagal pode depender do condicionamento aeróbio, e do tipo de treinamento realizado, além do índice de VFC utilizado na avaliaçáo.

Os determinantes autonômicos envolvidos na recuperação da FC pós-exercício, devido a coordenada reativação parassimpática e retirada simpática, são mediados pelos mecanismos de controle cardiovascular, como comando central, mecanorreflexo, metaboreflexo, atividade dos barorreceptores arterias e termorregulação ${ }^{21}$.

A dinâmica da FC e VFC sáo influenciados principalmente pela intensidade e tempo do exercício $^{22}$. Desta forma, o exercício aeróbico de alta intensidade, comparado ao exercício moderado e a sessão sem exercício físico, promove maior perturbação autonômica durante a recuperação, que pode se manter por várias horas pós-exercício em jovens sedentários não obesos ${ }^{23}$. A manutenção da inibição vagal após o exercício intenso deve-se a maior produção e liberação de metabólitos, como lactato, $\mathrm{H}^{+}, \mathrm{Pi}, \mathrm{K}^{+21}$ e hiperatividade simpática, que pode atrasar a reativação vagal cardíaca, reduzindo o decréscimo da FC na recuperação ${ }^{15,23}$. Foi demostrado, em modelos animais, que as elevadas concentraçóes de catecolaminas circulantes, sobretudo, norepinefrina, atenuam a estimulação vagal cardíaca devido a ativação dos receptores alfaadrenérgicos que reduz a liberação de acetilcolina, e influenciam a interação simpato-vagal ${ }^{24}$.

A reativação vagal foi verificada em jovens não atletas com aumento gradativo do índice RMSSD na recuperação passiva de 3 minutos após teste máximo ${ }^{25}$ e 30 minutos $^{26}$ pós-exercício a $70 \%$ da potência máxima, porém sem retorno aos valores de repouso ${ }^{25,26}$, sendo a reativação dependente da modalidade de exercício, pois o ciclismo apresentou maior aumento do RMSSD do que a corrida e a caminhada ${ }^{25}$. Contudo, a reativação completa da modulação vagal, pelo índice RMSSD, ocorreu após a recuperaçấo passiva de teste progressivo submáximo de menor intensidade $\left(50 \% \mathrm{VO}_{2 \text { max }}\right)$, aos 24,8 e 9,5 minutos em média, para os níveis de baixa e alta aptidão aeróbia, respectivamente ${ }^{27}$, demonstrando que os indivíduos com melhor aptidão aeróbia apresentam antecipadamente a re-estabilização no balanço simpático-vagal de repouso ${ }^{28}$. Corroborando nossos resultados, nos quais os grupos eutrófico e sobrepesos não apresentaram reativação vagal na recuperação ativa após exercício máximo, justificada pelos valores de $\mathrm{VO}_{2 \text { máx }}$ dos avaliados serem inferiores aos dos estudos mencionados ${ }^{28}$, assim como, o tempo de recuperação pós-exercício avaliado. 
Desse modo, o tipo, intensidade, duração do exercício, protocolo de recuperação, população estudada e nível de aptidão aeróbia são determinantes nas respostas da modulação autonômica cardíaca pós-exercício físico.

Houve associaçáo dos índices de VFC em repouso e na recuperaçáo, indicando que uma menor atividade parassimpática em repouso está associada a uma lenta recuperação pós-exercício. Contrariamente, os índices de repouso não se associaram à recuperação em jovens eutróficos sedentários e com maior $\mathrm{VO}_{2 \text { max }}{ }^{16}$. Sugere-se que se o presente grupo fosse constituído apenas por sujeitos obesos, esta tendência se confirmaria, uma vez que, o aumento da massa corporal está associado a disfunçóes autonômicas cardíacas, contudo, estas alteraçóes podem apenas se instalar na obesidade, que constituiu $55,5 \%$ dos avaliados com excesso de massa corporal.

A recuperação da FC após o exercício físico é um fator de risco modificável, com redução ponderal, que promove aumento da atividade vagal cardíaco, verificado em homens sobrepesos e obesos sem doença cardiovascular ${ }^{29}$. Em nosso estudo, o $\mathrm{VO}_{2 \text { max }}$ associouse significativa e inversamente à massa corporal e IMC. Além disso, a FC de repouso associou-se significativamente aos índices RMSSD e SDNN de repouso, com a área sob a curva do LnSDNN.

A prática de exercício físico promove melhora na capacidade aeróbia, no sistema cardiovascular e autonômico, além disso, permite a redução de diversos fatores de risco para doenças cardiovasculares ${ }^{3,11,19,25}$. Entretanto, o exercício físico é um estímulo estressor e perturba a modulação autonômica cardíaca, proporcionando aos indivíduos maior exposiçáo a riscos cardiovasculares, o que Albert et al..$^{30}$ denominaram de "janela de exposição", o período durante e logo após a realização de exercícios físicos em que o indivíduo fica exposto a maiores riscos cardiovasculares, os quais cessam apenas após o reestabelecimento do balanço simpato-vagal cardíaco de repouso. Neste sentido, a recuperação mais lenta após o exercício físico representa uma maior janela de exposição e consequentemente, maior risco cardiovascular ${ }^{12-14,30}$. No presente estudo verificouse que as variáveis de recuperação $\mathrm{FC}_{30 \mathrm{rec}}, \mathrm{FC}_{60 \mathrm{rec}}$ associaram-se negativamente às áreas sob a curva dos índices LnRMSSD e LnSDNN da VFC, indicando maior trabalho cardiovascular nesses jovens.

Dentre as limitaçóes do presente estudo, está a utilização do IPAQ para determinar o nível de atividade física, pois apesar de ser um questionário válido para a população deste estudo, não permite expressar de maneira mais apurada o gasto energético das atividades físicas como mediante o uso do acelerômetro. Assim como, a predição do $\mathrm{VO}_{2 \text { máx }}$ por equaçóes matemáticas, ainda que seja reprodutível e de baixo custo, apresenta erros de estimativa, enquanto a análise de gases expirados, medida direta do consumo de oxigênio, determinaria a capacidade aeróbia de maneira mais precisa.

Em suma, não há reativação vagal após 5 minutos de recuperação ativa de um exercício máximo em jovens, com comportamento similar entre os eutróficos e sobrepesos. Além disso, as variáveis morfológicas associam-se inversamente à aptidão aeróbia, e a FC aos índices de VFC nos diferentes momentos fisiológicos.

\section{Abstract}

\section{Heart rate variability in the recovery after maximal exercise in young overweight}

The aimed this study was analyze the heart rate variability (HRV) in the active recovery in young with different body mass index (BMI) and it was associate the variables at rest and recovery. Eighteen young, eutrophic - E $\left(22.36 \pm 1.92 \mathrm{~kg} \cdot \mathrm{m}^{-2}\right)$ and overweight $-0\left(29.71 \pm 2.97 \mathrm{~kg} \cdot \mathrm{m}^{-2}\right)$ were assessed. The heart rate $(\mathrm{HR})$ and the HRV index (RMSSD and SDNN) at rest, exercise and recovery after maximal exercise during 5 minutes, even as, the areas under the curve of the HRV were analyzed. The maximal oxygen uptake $\mathrm{VO}_{2 \text { máx }}$ was lower $(p<0.05)$ in the young overweight. The $\operatorname{LnRMSSD}_{30 \text { s }}$ and $\operatorname{LnSDNN}_{30 \mathrm{~s}}$ index at the end of the exercise ( $\mathrm{E}: 0.46 \pm 0.11$ and $0.75 \pm 0.12 ; 0: 0.58 \pm 1.15$ and $0.73 \pm 0.12 \mathrm{~ms})$ were reduced compared to rest ( $\mathrm{E}: 1.56 \pm 0.11$ and $1.72 \pm 0.13 ; 0: 1.42 \pm 0.11$ and $1.65 \pm 0.23 \mathrm{~ms})$ and have remained lower $(\mathrm{p}<0.05)$ at the end of the recovery ( $\mathrm{E}: 0.54 \pm 0.23$ and $0.67 \pm 0.24 ; 0: 0.47 \pm 0.23$ and $0.67 \pm 0.23 \mathrm{~ms}$ ) without differences between groups. Moreover, the morphologic variables were associated $(p<0.05)$ to aerobic fitness ( $r=-0.71$ to -0.76$)$, and the HR to HRV index in the different physiological moments. In conclusion, there was not vagal reactivation after 5 minutes of active recovery after a maximal exercise in young, with 
similar behavior between eutrophic and overweight. Also, morphological variables was associated inversely with the aerobic fitness, and the HR to HRV index in the different physiological moments.

KEYWORDS: Obesity; Cardiac autonomic modulation; Aerobic fitness.

\section{Agradecimentos}

Os autores agradecem à Fundação de Amparo à Pesquisa do Estado de Mato Grosso (Processo: 151411/2014), Conselho Nacional de Desenvolvimento Cientifico e Tecnológico, Pró-Reitoria de Pesquisa (PROPeq) pelo suporte financeiro.

\section{Conflito de interesse}

Os autores declaram não haver conflito de interesses

\section{Referências}

1. Singh JP, Larson MG, Tsuji H, Evans JC, O’Donnell CJ, Levy D. Reduced heart rate variability and newonset hypertension: insights into pathogenesis of hypertension: the Framingham Heart Study. Hypertension. 1998;32(2):293-7.

2. Jarczok MN, Li J, Mauss D, Fischer JE, Thayer JF. Heart rate variability is associated with glycemic status after controlling for components of the metabolic syndrome. Int J Cardiol. 2013;167(3):855-61.

3. Tulppo MP, Mäkikallio TH, Seppänen T, Laukkanen RT, Huikuri HV. Vagal modulation of heart rate during exercise: effects of age and physical fitness. Am J Physiol. 1998;274(2 Pt 2):H424-9.

4. Vanderlei LCM, Pastre CM, Hoshi RA, Carvalho TD, Godoy MF. Basic notions of heart rate variability and its clinical applicability. Rev Bras Cir Cardiovasc. 2009;24(2):205-17.

5. Laederach-Hofmann K, Mussgay L, Rúddel H. Autonomic cardiovascular regulation in obesity. J Endocrinol. 2000;164(1):59-66.

6. Kaye DM, Esler MD. Autonomic control of the aging heart. Neuromolecular Med. 2008;10(3):179-86.

7. Trevizani GA, Benchimol-Barbosa PR, Nadal J. Effects of age and aerobic fitness on heart rate recovery in adult men. Arq Bras Cardiol. 2012;99(3):802-10.

8. Barak OF, Jakovljevic DG, Gacesa JZP, Ovcin ZB, Brodie DA, Grujic NG. Heart rate variability before and after cycle exercise in relation to different body positions. J Sports Sci Med. 2010;9(2):176-82.

9. Arduini A, Gomez-Cabrera MC, Romagnoli M. Reliability of different models to assess heart rate recovery after submaximal bicycle exercise. J Sci Med Sport. 2011;14(4):352-7.

10. Dupuy O, Mekary S, Berryman N, Bherer L, Audiffren M, Bosquet L. Reliability of heart rate measures used to assess post-exercise parasympathetic reactivation. Clin Physiol Funct Imaging. 2012;32(4):296-304.

11. Yawn BP, Ammar KA, Thomas R, Wollan PC. Test-retest reproducibility of heart rate recovery after treadmill exercise. Ann Fam Med. 2003;1(4):236-41.

12. Cole CR, Blackstone EH, Pashkow FJ, Snader CE, Lauer MS. Heart-rate recovery immediately after exercise as a predictor of mortality. N Engl J Med. 1999;341(18):1351-7.

13. Cole CR, Foody JM, Blackstone EH, Lauer MS. Heart rate recovery after submaximal exercise testing as a predictor of mortality in a cardiovascularly healthy cohort. Ann Intern Med. 2000;132(7):552-5.

14. Nishime EO, Cole CR, Blackstone EH, Pashkow FJ, Lauer MS. Heart rate recovery and treadmill exercise score as predictors of mortality in patients referred for exercise ECG. JAMA. 2000;284(11):1392-8.

15. de Oliveira TP, de Alvarenga Mattos R, da Silva RB, Rezende RA, de Lima JR. Absence of parasympathetic reactivation after maximal exercise. Clin Physiol Funct Imaging. 2013;33(2):143-9. 
16. Guerra ZF, Peçanha T, Moreira DN, Silva LP, Laterza MC, Nakamura FY, Lima JR. Effects of load and type of physical training on resting and postexercise cardiac autonomic control. Clin Physiol Funct Imaging. 2014;34(2):114-20.

17. Vanderlei LCM, Pastre CM, Freitas Junior IF, Godoy MF. Analysis of cardiac autonomic modulation in obese and eutrophic children. Clinics (São Paulo). 2010;65(8):789-92.

18. Rossi RC, Vanderlei LC, Gonçalves AC, Vanderlei FM, Bernardo AF, Yamada KM, et al. Impact of obesity on autonomic modulation, heart rate and blood pressure in obese young people. Auton Neurosci. 2015;193:138-41.

19. D’Agosto T, Peçanha T, Bartels R, Moreira D, Silva L, Nóbrega A, Lima JR. Cardiac autonomic responses at onset of exercise: effects of aerobic fitness. Int J Sports Med. 2014;35(10):879-85.

20. Kokkinos P, Myers J, Doumas M, Faselis C, Pittaras A, Manolis A, et al. Heart rate recovery, exercise capacity, and mortality risk in male veterans. Eur J Prev Cardiol. 2012;19(2):177-84.

21. Pecanha T, Silva-Junior ND, Forjaz CL. Heart rate recovery: autonomic determinants, methods of assessment and association with mortality and cardiovascular diseases. Clin Physiol Funct Imaging. 2014;34(5):327-39.

22. Lucini D, Vigo C, Tosi F, Toninelli G, Badilini F, Pagani M. Assessing autonomic response to repeated bouts of exercise below and above respiratory threshold: insight from dynamic analysis of RR variability. Eur J Appl Physiol. 2014;114(6):1269-79.

23. Peçanha T, Prodel E, Bartels R, Nasario-Junior O, Paula RB, Silva LP, et al. 24-h cardiac autonomic profile after exercise in sedentary subjects. Int J Sports Med. 2014;35(3):245-52.

24. Miyamoto T, Kawada T, Takaki H, Inagaki M, Yanagiya Y, Jin Y, et al. High plasma norepinephrine attenuates the dynamic heart rate response to vagal stimulation. Am J Physiol Heart Circ Physiol. 2003;284(6):H2412-8.

25. Cunha FA, Midgley AW, Gonçalves T, Soares PP, Farinatti P. Parasympathetic reactivation after maximal CPET depends on exercise modality and resting vagal activity in healthy men. Springerplus. 2015;4:100.

26. Javorka M, Zila I, Balhárek T, Javorka K. Heart rate recovery after exercise: relations to heart rate variability and complexity. Braz J Med Biol Res. 2002;35(8):991-1000.

27. de Oliveira TP, de Lima JRP. Post-exercise cardiac full vagal reactivation: initial proposal and influence of physical training. J Exerc Physiol Online. 2012;15(6):103-11.

28. Hautala A, Tulppo MP, Mäkikallio TH, Laukkanen R, Nissilä S, Huikuri HV. Changes in cardiac autonomic regulation after prolonged maximal exercise. Clin Physiol. 2001;21(2):238-45.

29. Brinkworth GD, Noakes M, Buckley JD, Clifton PM. Weight loss improves heart rate recovery in overweight and obese men with features of the metabolic syndrome. Am Heart J. 2006;152(4):693.e1-6.

30. Albert CM, Mittleman MA, Chae CU, Lee IM, Hennekens CH, Manson JE. Triggering of sudden death from cardiac causes by vigorous exertion. N Engl J Med. 2000;343(19):1355-61.

\begin{tabular}{r|r} 
ENDEREÇO & \\
Gabriel Kolesny Tricot & \\
Departamento de Educação Física & Recebido para publicação: 23/o3/2016 \\
Universidade Federal de Mato Grosso & 1a. Revisão: 16/o6/2016 \\
Av. Fernando Corrêa da Costa, no 2367 & 2a. Revisão: 14/11/2016 \\
Boa Esperança & Aceito: 02/12/2016 \\
78060-90o - Cuiabá - MT - BRASIL & \\
e-mail: gabrielktricot.ufmt@gmail.com &
\end{tabular}

